\title{
Computational framework for particle and spin simulations based on the stochastic Galerkin method
}

\author{
J. Slim, ${ }^{1}$ F. Rathmann, ${ }^{2, *}$ and D. Heberling ${ }^{1,3}$ \\ ${ }^{1}$ Institut für Hochfrequenztechnik, RWTH Aachen University, 52056 Aachen, Germany \\ ${ }^{2}$ Institut für Kernphysik, Forschungszentrum Jülich, 52425 Jülich, Germany \\ ${ }^{3}$ JARA-FAME (Forces and Matter Experiments), Forschungszentrum Jülich and RWTH Aachen University, Aachen, Germany
}

(Received 3 September 2017; published 4 December 2017)

\begin{abstract}
An implementation of the polynomial chaos expansion is introduced as a fast solver of the equations of beam and spin motion of charged particles in electromagnetic fields. We show that, based on the stochastic Galerkin method, our computational framework substantially reduces the required number of tracking calculations compared to the widely used Monte Carlo method.
\end{abstract}

DOI: 10.1103/PhysRevE.96.063301

\section{INTRODUCTION AND MOTIVATION}

The aim of the $\mathrm{JEDI}^{1}$ collaboration is to measure the electric dipole moment (EDM) of charged hadronic particles, such as deuterons and protons. In the near future, a first direct EDM measurement of the deuteron [1,2] will be carried out at the COoler SYnchrotron COSY [3,4]. Toward that end, an RF Wien filter [5] has recently been installed, which is operated at some harmonic of the spin precession frequency, whereby the sensitivity to the deuteron EDM is substantially enhanced.

To eliminate false EDM signals, it is of crucial importance to understand the sources of imperfections in the accelerator ring that is used to perform the measurements [6]. Particle and spin tracking simulations constitute powerful tools to accomplish this $[7,8]$. The realistic simulation of a precision physics experiment in a storage ring [9] that includes also mechanical and electromagnetic uncertainties in all of its elements, however, represents a very challenging task.

Monte Carlo (MC) simulations as a tool to evaluate the systematics of a precision experiment are computationally very expensive, and the dimension (the number of random parameters) in such simulations is particularly large. These simulations usually have to be repeated many times in order to obtain small uncertainties of the parameters under study. The use of the stochastic Galerkin method (SGM) is motivated by the requirement to replace the computer-expensive MC method by a more efficient computational technique, which transforms a system of differential equations that describe the quantities of interest into an augmented system of equations that contains only the coefficients [10]. It should be noted that the SGM does not alter the intrinsic properties of the solver; in fact, the SGM considers the solver as a black box.

To provide a more efficient simulation framework that avoids the computationally expensive MC simulations, and as a first step toward a fully systematic analysis of the future EDM experiments at COSY, we recently conducted a study to quantify the electromagnetic performance of the above-mentioned RF Wien filter, taking into account various

\footnotetext{
*Author to whom all correspondence should be addressed: f.rathmann@fz-juelich.de

${ }^{1}$ Jülich Electric Dipole moment Investigations, http://collaborations.fz-juelich.de/ikp/jedi/
}

mechanical uncertainties [11]. This investigation made use of the so-called polynomial chaos expansion (PCE) [12] as an efficient and yet accurate alternative to the MC method.

In this paper, we describe a computational framework based on the stochastic Galerkin method to determine the solutions of the equations of beam and spin motion. This approach substantially reduces the required number of tracking simulations, but we still arrive at the same result as that of a standard MC simulation. The methodology used is similar to the one described in Ref. [11], but since we have access to the equations of motion and spin governing the system, an intrusive version of the PCE is employed.

The paper is organized as follows. The basic theoretical foundations of the PCE are briefly reviewed in Sec. II, and in Sec. III the SGM is introduced and applied to the beam and spin equations. The main steps to perform the SGM are described in Sec. IV. In Sec. V, the results obtained for a uniform electromagnetic field and the RF field of an RF Wien filter are compared quantitatively to the equivalent $\mathrm{MC}$ results. The conclusions are presented in Sec. VI.

\section{POLYNOMIAL CHAOS EXPANSION}

The polynomial chaos expansion (PCE) is a stochastic spectral method that allows for stochastically varying physical entities $\mathcal{Y}$, as a response of some random input $\xi$ to be represented in terms of orthogonal polynomials. The PCE permits $\mathcal{Y}$ to be expanded into a series of orthogonal polynomials of degree $p$ (the expansion order) as a function of the input variables $\xi$. Thus it follows that

$$
\mathcal{Y}=\sum_{i}^{N} a_{i} \Psi_{i}(\xi) .
$$

Here $\Psi_{i}$ are the multivariate orthogonal polynomials (the basis functions) of degree $p$, and $a_{i}$ are the expansion coefficients to be computed. The orthogonal polynomials can be the Hermite, Legendre, Laguerre, or any other set of orthogonal polynomials, depending on the probabilistic distribution of the random input variables $\xi$. The physical entities $\mathcal{Y}$ include electromagnetic fields (with uncertainties), particle positions, velocities, and spin vectors.

The first step in building a polynomial chaos series is to determine the probabilistic distribution of the input random 
variables and their number (the dimensionality of the problem). Once known, the multivariate orthogonal polynomials can be constructed using the three-term recurrence properties that can be found, e.g., in [13].

In multidimensional problems, multivariate polynomials are used to build the chaos basis. Obviously, the expansion order must be known, and, as explained in [14,15], it can be changed adaptively during processing.

The expansion coefficients can be calculated using intrusive and nonintrusive methods. Nonintrusive methods consider the deterministic code as a black box, i.e., they do not alter the code or the equations. The expansion coefficients are calculated using multiple calls to the deterministic code either via projection or regression. Both require a number of $N$ realization pairs $(\xi, \mathcal{Y})$ [see Eq. (1)], and they are further elucidated below.

Projection requires the evaluation of expectation values and relies on the orthogonality of the polynomials to compute the coefficients in the form of

$$
a=\frac{\mathbb{E}\{\mathcal{Y} \Psi\}}{\mathbb{E}\left\{\Psi^{2}\right\}} .
$$

The computation of the expectation values $(\mathbb{E}\{\cdot\})$ necessitates the evaluation of integrals. Quadrature methods are one way to do so, and they are commonly used in PCE analyses. Depending on the type of input distribution, the corresponding quadrature rule can be used. The Gauss-Laguerre quadrature, for instance [16], is used in the case of uniformly distributed random variables. It is widely known as nonintrusive spectral projection (NISP) [17].

Regression, on the other hand, estimates the coefficients that minimize the functional difference between the estimated response $\hat{\mathcal{Y}}$ and the actual response $\mathcal{Y}$, given by

$$
a=\arg \min \left(\mathbb{E}\{\hat{\mathcal{Y}}-\mathcal{Y}\}^{2}\right) .
$$

The solution of Eq. (3), obtained by linear regression, yields

$$
a_{i}=\left(\Psi^{T} \cdot \Psi\right) \cdot \Psi \cdot \mathcal{Y} .
$$

In the context of the work presented in this paper, the regression method [18] provides more accurate results than the projection method, and it is therefore used here to calculate the expansion coefficients $a_{i}$, which are subsequently used as an input for the stochastic Galerkin solver.

\section{STOCHASTIC GALERKIN METHOD APPLIED TO BEAM AND SPIN DYNAMICS}

\section{A. Beam dynamics}

One of the most common methods to solve differential equations is the Galerkin finite-element method (FEM) $[19,20]$. In 1921, the Russian mathematician Boris Galerkin proposed a method to solve differential equations based on functional analysis. In contrast to other methods, such as finitedifference (FD) schemes, the Galerkin method does not solve the differential equations directly, rather it transforms them into a variational form (a functional) that is then minimized. The functions minimizing this functional are the solutions to the required differential equations. The variational form is constructed via the Galerkin projection techniques [17].

In this section, the construction of the variational form of the beam and spin dynamic equations using the stochastic Galerkin projection is described in detail. Neglecting forces other than the electromagnetic ones acting on the charged particles, the beam equations read $[7,21]$

$$
\begin{aligned}
\frac{d}{d t} \vec{v} & =\frac{q}{m \gamma}\left[\vec{E}+\vec{v} \times \vec{B}-\frac{1}{c^{2}} \vec{v}(\vec{v} \cdot \vec{E})\right], \\
\frac{d}{d t} \vec{r} & =\vec{v} .
\end{aligned}
$$

Here, $\vec{E}$ and $\vec{B}$ represent the electric and magnetic fields, $\vec{v}$ denotes the velocity vector of the particles, $q$ is the particle charge, $m$ is the mass, $\gamma$ is the Lorentz factor, $\vec{r}$ is the position vector, and $\vec{v}$ is the velocity vector of the particles.

The expansion of Eq. (5) in Cartesian coordinates yields a linear system of six coupled ordinary differential equations,

$$
\begin{aligned}
\frac{d}{d t} v_{x} & =\frac{q}{m}\left[\frac{1}{\gamma} E_{x}+\frac{1}{\gamma} v_{y} B_{z}-\frac{1}{\gamma} v_{z} B_{y}-\frac{1}{c^{2} \gamma} v_{x}(\vec{v} \cdot \vec{E})\right], \\
\frac{d}{d t} v_{y} & =\frac{q}{m}\left[\frac{1}{\gamma} E_{y}+\frac{1}{\gamma} v_{z} B_{x}-\frac{1}{\gamma} v_{x} B_{z}-\frac{1}{c^{2} \gamma} v_{y}(\vec{v} \cdot \vec{E})\right], \\
\frac{d}{d t} v_{z} & =\frac{q}{m}\left[\frac{1}{\gamma} E_{z}+\frac{1}{\gamma} v_{x} B_{y}-\frac{1}{\gamma} v_{y} B_{x}-\frac{1}{c^{2} \gamma} v_{z}(\vec{v} \cdot \vec{E})\right], \\
\frac{d}{d t} x & =v_{x}, \\
\frac{d}{d t} y & =v_{y}, \\
\frac{d}{d t} z & =v_{z} .
\end{aligned}
$$

When intrabeam scattering and other collective effects are neglected, the simulation of a beam of particles is equivalent to individual simulations with different initial conditions. It is furthermore assumed that the number of single-particle simulations is sufficiently large to describe the beam. These assumptions permit us to use stochastic methods to solve the differential equations with random coefficients, or with uncertain input variables, or even with random boundary values. According to a probabilistic distribution, each individual particle of the population has a different initial position $\vec{r}$ and velocity vector $\vec{v}$. Therefore, the treatment of these parameters as random vectors in Eq. (5) justifies the application of the SGM.

Equation (5) describes an initial value problem, where the initial values vary randomly. The initial values are expanded using nonintrusive PC, particularly with linear regressions, and then the SGM [22] is applied to solve Eq. (5). As an example, the technique of solving for the variable $v_{x}$ is discussed in detail below. 
$v_{x}$ is expanded as

$$
v_{x}=\sum_{i}^{N} v_{x_{i}}^{(k)} \Psi_{i}
$$

where the $v_{x_{i}}{ }^{(k)}$ are the chaos expansion coefficients. The superscript $(k)$ is used to identify the expansion coefficients, and also to emphasize that the variables are discretized. The coefficients are calculated according to

$$
v_{x_{i}}{ }^{(k)}=\left(\Psi^{T} \cdot \Psi\right) \cdot \Psi \cdot v_{x_{0}},
$$

where $v_{x_{0}}$ are the initial $x$-components of the particle velocities. Inserting Eq. (7) into the left-hand side of Eq. (6a), we find

$$
\frac{d}{d t} v_{x}=\frac{d}{d t} \sum_{i}^{N} v_{x_{i}}^{(k)} \Psi_{i}=\sum_{i}^{N} \frac{d}{d t} v_{x_{i}}^{(k)} \Psi_{i} .
$$

Now, the stochastic Galerkin projection is applied by multiplying Eq. (9) with $\Psi_{l}$ and taking the expectation value $\mathbb{E}\{\cdot\}$, which gives

$$
\begin{aligned}
\mathbb{E}\left\{\sum_{i}^{N} \frac{d}{d t} v_{x_{i}}{ }^{(k)} \Psi_{i} \Psi_{l}\right\} & =\sum_{i}^{N} \frac{d}{d t} v_{x_{i}}^{\left({ }^{k}\right)} \mathbb{E}\left\{\Psi_{i} \Psi_{l}\right\} \\
& =\sum_{i}^{N} \frac{d}{d t} v_{x_{i}}^{(k)}\left\langle\Psi_{i} \Psi_{l}\right\rangle \\
& =\sum_{i}^{N} \frac{d}{d t} v_{x_{i}}^{(k)}\left\langle\Psi_{i}^{2}\right\rangle \delta_{i l} .
\end{aligned}
$$

Here $\delta_{i l}$ is the Kronecker delta, which results from the orthogonality of the polynomials.

The electric field is also represented stochastically ${ }^{2}$ by the finite series

$$
E_{x}=\sum_{i}^{N} e_{x_{i}}^{(k)} \Psi_{i}
$$

The Lorentz factor $\gamma$ constitutes also a stochastic variable. Unfortunately, it appears in the denominator of all terms in Eq. (5). To solve this problem, $1 / \gamma$ is expanded instead of $\gamma$. Let $\alpha$ be defined as

$$
\alpha=\frac{1}{\gamma}
$$

Then $\alpha$ is expanded as

$$
\alpha=\sum_{i}^{N} \alpha_{i}^{(k)} \Psi_{i}
$$

The stochastic Galerkin projection is applied by multiplying the product of Eqs. (11) and (13) by $\Psi_{k}$, and subsequently

\footnotetext{
${ }^{2}$ The Cartesian components of the electric and magnetic fields $(\vec{E}$ and $\vec{B})$ are functions of the position vector $\vec{r}$, e.g., $\vec{E}(\vec{r}), \vec{B}(\vec{r})$, etc. The dependence of the field components on position, e.g., $E_{x}(\vec{r})=$ $E_{x}(x, y, z)$, does not pose a problem for the PCE method as long as the input variables (e.g., $r$ and $v$ ) are independent.
}

calculating the expectation value $\mathbb{E}\{\cdot\}$. It thus follows that

$$
\begin{aligned}
\mathbb{E}\left\{\sum_{i}^{N} e_{x_{i}}{ }^{(k)} \Psi_{i} \sum_{j}^{N} \alpha_{j}^{(k)} \Psi_{j} \Psi_{l}\right\} & =\sum_{i}^{N} \sum_{j}^{N} e_{x_{i}}^{(k)} \alpha_{j}^{(k)}\left\langle\Psi_{i} \Psi_{j} \Psi_{l}\right\rangle \\
& =\sum_{i}^{N} \sum_{j}^{N} \alpha_{i}^{(k)} e_{x_{j}}^{(k)} C_{i j l} .
\end{aligned}
$$

The $C_{i j l}=\left\langle\Psi_{i} \Psi_{j} \Psi_{l}\right\rangle$ tensor constitutes a sparse rank-3 tensor. It is constructed offline by computing the tensor product, which constitutes a CPU-intensive operation. The formula to compute $C_{i j l}$ is provided in [22], and it works only for low-order and low-dimensional cases. In addition, it is limited to Gaussian-distributed random variables, and consequently it applies only to Hermite polynomials. For the actual version implemented here, $C_{i j l}$ is computed numerically and is distribution-independent. The implementation has been validated with a one-dimensional quadrature-based one and yielded the same results.

Fortunately, $C_{i j l}$ needs to be computed only once. It can be stored and reused when required. Although the multiplications of the PCE coefficients involve the $C_{i j l}$ term, this arithmetic operation does not introduce any computational overhead as $C_{i j l}$ is sparse. This is illustrated in Fig. 1 for several typical examples of the $C_{i j l}$ tensor.

The next term of Eq. (6a), namely the product of $\alpha$, velocity $v_{y}$, and magnetic field $B_{z}$, presents a more complicated situation because it involves multiple polynomials. This triple product requires us to expand the two latter quantities as

$$
\begin{aligned}
& v_{y}=\sum_{i}^{N} v_{y_{i}}{ }^{(k)} \Psi_{i}, \\
& B_{z}=\sum_{i}^{N} b_{z_{i}}{ }^{(k)} \Psi_{i},
\end{aligned}
$$

where $v_{y_{i}}{ }^{(k)}$ and $b_{z_{i}}{ }^{(k)}$ are the expansion coefficients of $v_{y}$ and $B_{z}$, respectively. The multiplication of the three sums yields

$$
\alpha v_{y} B_{z}=\sum_{i}^{N} \sum_{j}^{N} \sum_{k}^{N} \alpha_{i}^{(k)} v_{y_{j}}^{(k)} b_{z k}^{(k)} \Psi_{i} \Psi_{j} \Psi_{k} .
$$

By applying the stochastic Galerkin projection to Eq. (16), it follows that

$$
\begin{aligned}
\mathbb{E}\left\{\alpha v_{y} B_{z} \Psi_{l}\right\} & =\sum_{i}^{N} \sum_{j}^{N} \sum_{k}^{N} \alpha_{i}^{(k)} v_{y_{j}}^{(k)} b_{z_{k}}^{(k)}\left\langle\Psi_{i} \Psi_{j} \Psi_{k} \Psi_{l}\right\rangle \\
& =\sum_{i}^{N} \sum_{j}^{N} \sum_{k}^{N} \alpha_{i}^{(k)} v_{y_{i}}^{(k)} b_{z_{j}}^{(k)} D_{i j k l}
\end{aligned}
$$

$D_{i j k l}$ is similar to $C_{i j l}$, but it constitutes a rank-4 tensor. The case for the third term of Eq. (6a) yields

$$
\begin{aligned}
\mathbb{E}\left\{\alpha v_{z} B_{y} \Psi_{k}\right\} & =\sum_{i}^{N} \sum_{j}^{N} \sum_{k}^{N} \alpha_{i}^{(k)} v_{z_{j}}^{(k)} b_{y_{k}}^{(k)}\left\langle\Psi_{i} \Psi_{j} \Psi_{k} \Psi_{l}\right\rangle \\
& =\sum_{i}^{N} \sum_{j}^{N} \sum_{k}^{N} \alpha_{i}^{(k)} v_{z_{j}}^{(k)} b_{y k}^{(k)} D_{i j k l} .
\end{aligned}
$$




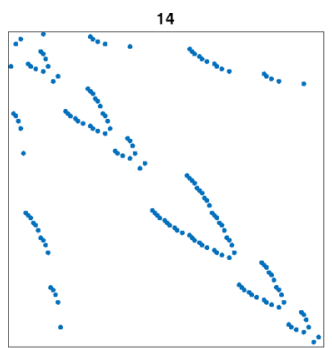

(a)

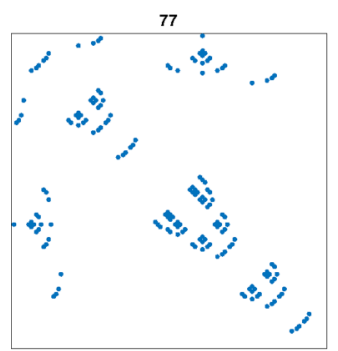

(f)

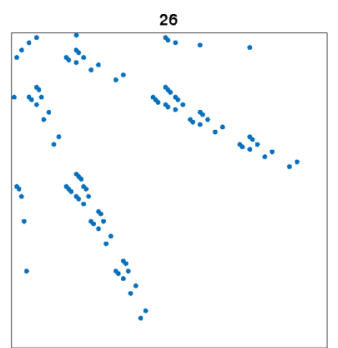

(b)

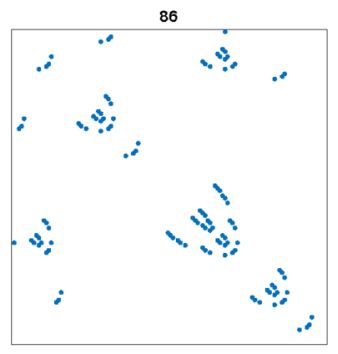

(g)

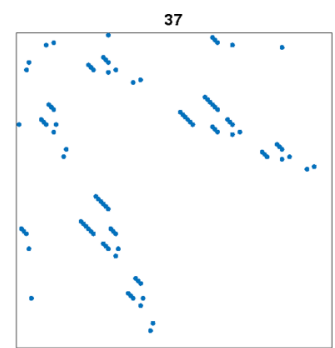

(c)

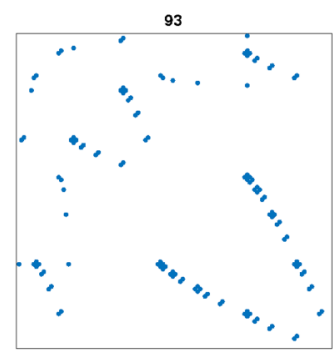

(h)

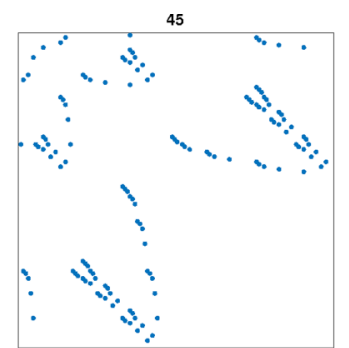

(d)

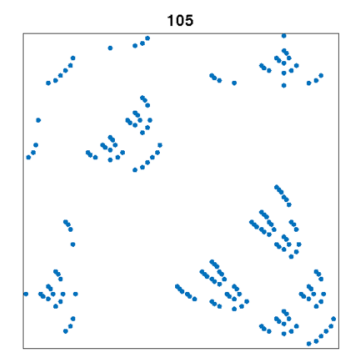

(i)

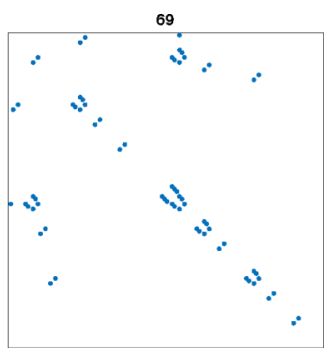

(e)

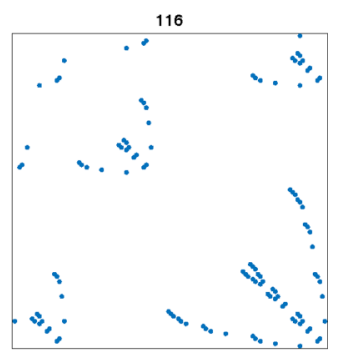

(j)

FIG. 1. With the $(m=5)$-dimensional problem and an expansion order of $p=4$, the number of basis functions is $P=126$ [see Eq. (7) of [11]], which results in a $(126 \times 126 \times 126) C_{i j l}$ tensor. The sparsity of the rank-3 tensor $C_{i j l}$ is illustrated by fixing, e.g., the first index to values of $i=14$ [panel (a)], $i=26$ (b), $i=37$ (c), $i=45$ (d), $i=69$ (e), $i=77$ (f), $i=86$ (g), $i=93$ (h), $i=105$ (i), and $i=116$ (j), which yields the 10 sparse matrices shown. The multiplication of the PCE coefficients involving this tensor is very fast, as most of the tensor elements are zero, while computing, storing, and loading of this tensor constitutes a CPU and memory-intensive operation.

The last term of the right-hand side of Eq. (6a), i.e.,

$$
\frac{1}{c^{2}} \alpha v_{x}(\vec{v} \cdot \vec{E})
$$

is even more complicated because it involves a scalar product. The scalar product operator multiplies the operands componentwise before summing them up. These operands, however, are PC coefficients. The corresponding multiplication is in fact a Galerkin one, which involves a series of double products [23], given by

$$
\vec{v} \cdot \vec{E}=\sum_{i}^{3} \sum_{j}^{N} \sum_{k}^{N} v_{i}^{(k)} e_{i}^{(k)} \Psi_{j} \Psi_{k}
$$

This means that Eq. (20) requires the stochastic Galerkin projection to compute a rank-5 tensor, which makes the method highly inefficient [23]. To solve this problem, a pseudospectral method $[17,24]$ is used. The Galerkin projection is applied first to the auxiliary variable $g_{l}$ (the one representing the scalar product) and then second to the full product in Eq. (19). This way, the rank-4 tensor product, introduced above in Eq. (17), can be used. In particular, $g_{l}$ reads

$$
g_{l}=\mathbb{E}\left\{(\vec{v} \cdot \vec{E}) \Psi_{l}\right\}=\sum_{i}^{3} \sum_{j}^{N} \sum_{k}^{N} v_{i j}^{(k)} e_{i}{ }_{k}^{(k)} C_{i j l},
$$

where the subscript $l$ here constitutes a free variable. And then, by applying the stochastic Galerkin projection, it follows that

$$
\mathbb{E}\left\{\alpha v_{x}(\vec{v} \cdot \vec{E}) \Psi_{l}\right\}=\sum_{i}^{N} \sum_{j}^{N} \sum_{k}^{N} \alpha_{i}^{(k)} v_{x}{ }_{j}^{(k)} g_{k}^{(k)} D_{i j k l} .
$$

The other five equations, Eqs. (6b)-(6f), are treated in a similar fashion, and the system of ordinary differential equations (ODEs) is considered solved.

\section{B. Spin dynamics}

The spin dynamics in an electromagnetic storage ring with nonvanishing EDM is described by the generalized T-BMT equation $[25,26]$, which reads

$$
\frac{d}{d t} \vec{S}=\left(\vec{\Omega}^{\mathrm{MDM}}+\vec{\Omega}^{\mathrm{EDM}}\right) \times \vec{S}
$$

Here, $\vec{S}$ denotes the particle spin, and $\vec{\Omega}^{\mathrm{EDM}}$ and $\vec{\Omega}^{\mathrm{MDM}}$ are the angular velocities associated with the magnetic (MDMs) and electric dipole moments (EDMs). $\vec{\Omega}^{\mathrm{MDM}}$ and $\vec{\Omega}^{\mathrm{EDM}}$ are defined as

$$
\begin{aligned}
\vec{\Omega}^{\mathrm{MDM}}= & -\frac{q}{m \gamma}\left[(1+G \gamma) \vec{B}+\left(G \gamma+\frac{\gamma}{1+\gamma}\right) \frac{\vec{E} \times \vec{\beta}}{c}\right. \\
& \left.-\frac{G \gamma^{2}}{\gamma+1} \vec{\beta}(\vec{\beta} \cdot \vec{B})\right], \\
\vec{\Omega}^{\mathrm{EDM}}= & -\frac{q}{m} \frac{\eta}{2}\left[\frac{\vec{E}}{c}+\vec{\beta} \times \vec{B}-\frac{\gamma}{\gamma+1} \vec{\beta}\left(\vec{\beta} \cdot \frac{\vec{E}}{c}\right)\right] .
\end{aligned}
$$

The particle velocity is given by $\vec{\beta}=\vec{v} / c, G$ denotes the anomalous magnetic moment, and $\eta$ is a dimensionless parameter, proportional to the particles' EDM. 
Expanding Eq. (23) in Cartesian coordinates reads

$$
\begin{aligned}
& \frac{d}{d t} S_{x}=\Omega_{y}^{\mathrm{MDM}} S_{z}-\Omega_{z}^{\mathrm{MDM}} S_{y}+\Omega_{y}^{\mathrm{EDM}} S_{z}-\Omega_{z}^{\mathrm{EDM}} S_{y}, \\
& \frac{d}{d t} S_{y}=\Omega_{z}^{\mathrm{MDM}} S_{x}-\Omega_{x}^{\mathrm{MDM}} S_{z}+\Omega_{z}^{\mathrm{EDM}} S_{x}-\Omega_{x}^{\mathrm{EDM}} S_{z}, \\
& \frac{d}{d t} S_{z}=\Omega_{x}^{\mathrm{MDM}} S_{y}-\Omega_{y}^{\mathrm{MDM}} S_{x}+\Omega_{x}^{\mathrm{EDM}} S_{y}-\Omega_{y}^{\mathrm{EDM}} S_{x} .
\end{aligned}
$$

Before proceeding to the stochastic discretization of the spin equation (23), some variables are introduced to simplify the discretization process. Let

$$
\begin{aligned}
& f_{1}=\frac{1}{\gamma}+G, \\
& f_{2}=\frac{1}{c}\left(G+\frac{1}{\gamma}\right), \\
& f_{3}=\frac{G \gamma}{1+\gamma}, \\
& f_{4}=\frac{\gamma}{\gamma+1} .
\end{aligned}
$$

All the terms that interact with field and velocity components are grouped together. It should be noted that here only $\gamma$ constitutes a stochastic variable. The PCE coefficients are also calculated using the nonintrusive projection method, yielding

$$
\begin{aligned}
& f_{1}=\sum_{i}^{N} f_{1_{i}} \Psi_{i}, \\
& f_{2}=\sum_{i}^{N} f_{2_{i}} \Psi_{i}, \\
& f_{3}=\sum_{i}^{N} f_{3 i} \Psi_{i}, \\
& f_{4}=\sum_{i}^{N} f_{4_{i}} \Psi_{i} .
\end{aligned}
$$

$\vec{\Omega}^{\mathrm{MDM}}$ and $\vec{\Omega}^{\mathrm{EDM}}$ are rewritten as

$$
\begin{aligned}
& \vec{\Omega}^{\mathrm{MDM}}=-\frac{q}{m}\left[f_{1} \vec{B}+f_{2}(\vec{E} \times \vec{\beta})-f_{3} \vec{\beta}(\vec{\beta} \cdot \vec{B})\right], \\
& \vec{\Omega}^{\mathrm{EDM}}=-\frac{q}{m} \frac{\eta}{2}\left[\frac{\vec{E}}{c}+\vec{\beta} \times \vec{B}-f_{4} \vec{\beta}\left(\vec{\beta} \cdot \frac{\vec{E}}{c}\right)\right] .
\end{aligned}
$$

In the following, the same methodology as described in Sec. III A is applied to the spin equation. By induction from the derivation described above, beginning with the $x$ component of the spin vector in Eq. (24), it follows that

$$
\begin{aligned}
\sum_{i}^{N} \frac{d}{d t} S_{x}{ }^{(k)}= & \sum_{i}^{N} \sum_{j}^{N} \Omega_{y}^{\mathrm{MDM}_{i}^{(k)}} S_{z j}^{(k)} C_{i j l} \\
& -\sum_{i}^{N} \sum_{j}^{N} \Omega_{z}^{\mathrm{MDM}_{i}^{(k)}} S_{y_{j}}^{(k)} C_{i j l}
\end{aligned}
$$

$$
\begin{aligned}
& +\sum_{i}^{N} \sum_{j}^{N} \Omega_{y}^{\mathrm{EDM}_{i}^{(k)}} S_{z j}^{(k)} C_{i j l} \\
& -\sum_{i}^{N} \sum_{j}^{N} \Omega_{z}^{\mathrm{EDM}_{j}^{(k)}} S_{y_{j}}^{(k)} C_{i j l} .
\end{aligned}
$$

Rewriting, e.g., $\Omega_{y}^{\mathrm{MDM}_{i}^{(k)}}$ in terms of the individual components is equivalent to the following expression:

$$
\begin{aligned}
& \sum_{i}^{N} \Omega_{y}^{\mathrm{MDM}^{(k)}} \\
& =-\frac{q}{m}\left[\sum_{i}^{N} \sum_{j}{f_{1}}_{1_{i}}^{(k)} b_{y_{j}}{ }^{(k)} C_{i j l}\right. \\
& +\sum_{i}^{N} \sum_{j} \sum_{k}\left(f_{2_{i}}^{(k)} e_{z_{i}}^{(k)} \beta_{x_{j}}^{(k)} D_{i j k l}-f_{2_{i}}^{\left({ }^{k}\right)} e_{x_{i}}^{(k)} \beta_{z_{j}}^{(k)} D_{i j k l}\right) \\
& \left.-\sum_{i}^{N} \sum_{j}^{N} \sum_{k}^{N} f_{3_{i}}^{(k)} \beta_{y_{j}}^{(k)} h_{k}^{(k)} D_{i j k l}\right],
\end{aligned}
$$

where

$$
h_{l}=\mathbb{E}\left\{(\vec{\beta} \cdot \vec{B}) \Psi_{l}\right\}=\sum_{i}^{3} \sum_{j}^{N} \sum_{k}^{N} \beta_{i j}^{(k)} b_{i k}{ }^{(k)} C_{i j l} .
$$

Here, $l$ constitutes a free subscript [later on replaced by $k$ in Eq. (30)]. Similarly, the other components of the spin dynamics equation (23) can be constructed, but this derivation is omitted here for brevity.

\section{SIMULATIONS}

The stochastic Galerkin method (SGM) transforms a system of differential equations that describe the quantities of interest into an augmented system of equations that contains only the coefficients [10]. Depending on the dimension and the expansion order $p$, the dimension of the new system of equations is determined. The solutions of the system are called stochastic modes, which are merely the time- and position-dependent expansion coefficients that will later be used to reconstruct the response for an arbitrary number of particles.

To perform the simulations, the random variables have to be identified first. These include the particle positions, velocities, and spins. Next, the expansion order $p$ is selected with the smallest possible value in order for the augmented system of equations to be as small as possible as well. The expansion order can later be increased if the achieved accuracy appears to be unsatisfactory. In this work, the expansion order was set to $p=4$, which, as shown later, yields very small parameter errors.

Since Gaussian distributions were selected here, the basis functions are Hermite polynomials. The distributions of the random variables must be normalized in order for the SGM to converge. The total number of polynomials (cardinality of polynomials) is $P=126$ [11]. 


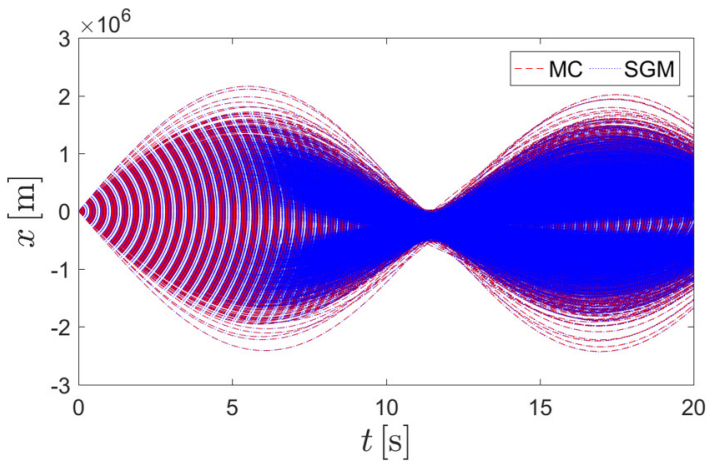

(a)

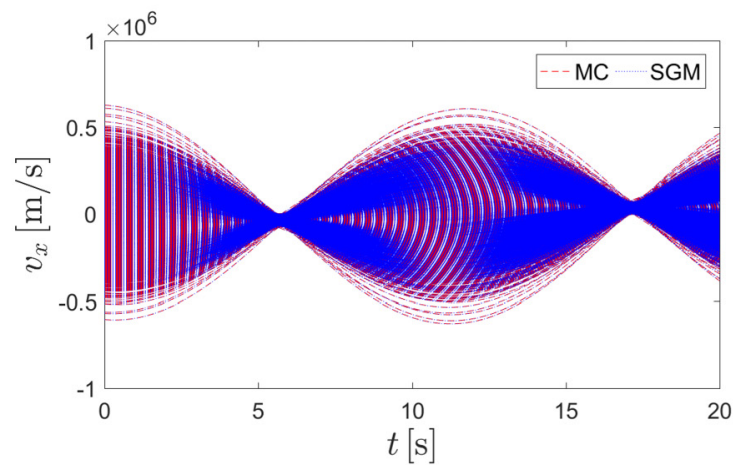

(c)

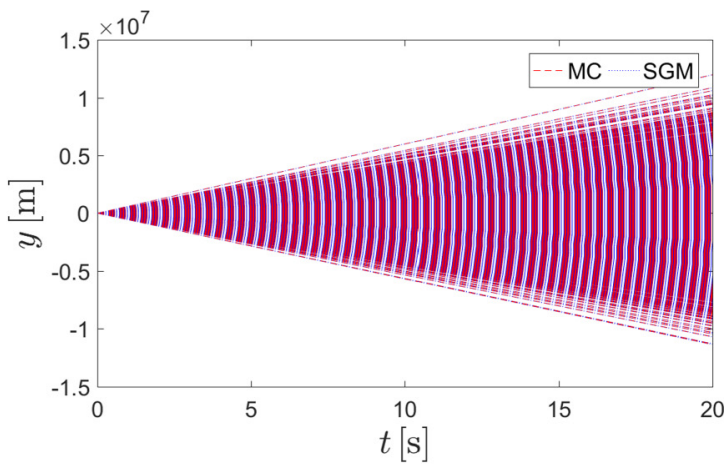

(b)

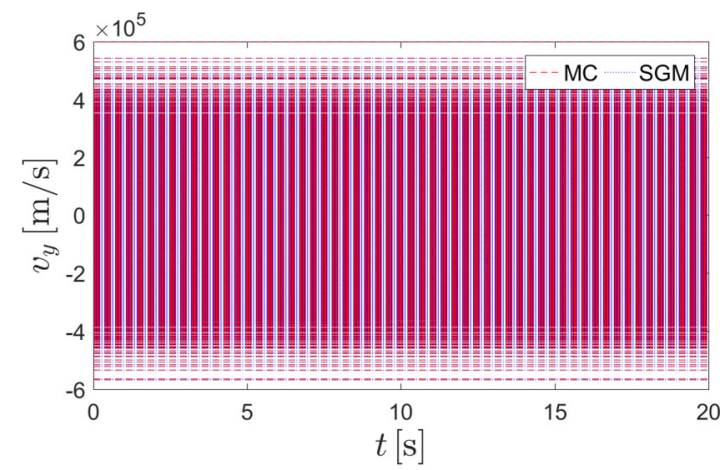

(d)

FIG. 2. Results of tracking $10^{5}$ particles in a uniform electric and magnetic field $[\vec{E}=(1,0,0) \mathrm{V} / \mathrm{m}, \vec{H}=(0,1 / 173,0)$ A/m]. On the horizontal axis, the time in seconds is shown over which the tracking simulation evolved, and on the vertical axis the resulting solutions for $x(t)$ in (a), $y(t)(\mathrm{b}), v_{x}(t)(\mathrm{c})$, and $v_{y}(t)(\mathrm{d})$. The dotted blue lines represent the solution computed using the Monte Carlo simulations (MC), while the dashed red lines visualize the solutions of the stochastic Galerkin method (SGM). In all four cases, positions and velocities are nearly indistinguishable, indicating a very good agreement between MC and SGM (see also Fig. 4).

The particles are generated according to well-defined phase-space distributions. An example is shown in Fig. 9 of [5]. Such populations might violate the independency requirement that the parameters be random. In this case, the Nataf transformation [27-29] ${ }^{3}$ can be used to circumvent the problem. With all these parameters, the expansion coefficients of the initial particle population are constructed using the linear regression method, as described in Eq. (8).

With $P=126, C_{i j l}$ is a $126 \times 26 \times 126$ tensor. Unless a new random quantity is added to the analysis, or the order of the expansion is changed, the stored $C_{i j l}$ can be used when required. When the expansion order $p$ or the dimension of the problem becomes large, pseudospectral methods may become more favorable (see, e.g., [30]).

The SGM-based system of equations has been solved using MATLAB. ${ }^{4,5}$ The deterministic ordinary differential equations

\footnotetext{
${ }^{3}$ Nataf transformations are isoprobabilistic transformations that transform correlated Gaussian variables with arbitrary mean and variance into normally distributed ones. Without such a transformation, the orthogonality of the basis functions would be violated.

${ }^{4}$ Mathworks, Inc. Natick, Massachusetts, United States http://www.mathworks.com

${ }^{5}$ The simulations were performed on a HP Z840 workstation with a single Xeon E5v4 CPU and a RAM capacity of $80 \mathrm{~GB}$.
}

solver "ode $45^{\prime \prime 6}$ was employed with a fixed time step of $1 \mathrm{~ms}$, and relative and absolute error tolerances of $10^{-13}$ and $10^{-20}$, respectively. It is important to note that the same ODE solver has been applied to both MC simulation and SGM calculation.

At the final stage, the performance of the SGM must be evaluated quantitatively, with the help of an adequate error analysis. Due to time and position dependencies, the error calculation involves either the mean value $(\mu)$ or the standard deviation $(\sigma)$ of the quantity under investigation, denoted in the following by $\zeta$. The corresponding errors are called $\epsilon_{\mu}$ and $\epsilon_{\sigma}$, respectively, and they are defined as

$$
\begin{aligned}
& \epsilon_{\mu}(t)=\left|\frac{\mu[\zeta(t)]-\mu[\hat{\zeta}(t)]}{\mu[\zeta(t)]}\right|, \quad \text { and } \\
& \epsilon_{\sigma}(t)=\left|\frac{\sigma[\zeta(t)]-\sigma[\hat{\zeta}(t)]}{\sigma[\zeta(t)]}\right| .
\end{aligned}
$$

Here, $\zeta$ may refer to either the position, velocity, or spin vector, while $\hat{\zeta}$ denotes the value estimated using the SGM. To conduct an analysis similar to the one described in [12,31], the exact initial conditions are inserted into both the $\mathrm{MC}$ and the SGM solver, so that the solutions can be directly compared on a particle-by-particle basis. In this way, the difference of

\footnotetext{
${ }^{6}$ The "ode45" solver is based on the fourth-order Runge-Kutta integration technique.
} 
TABLE I. Parameters of a deuteron beam stored at COSY at a momentum of $970 \mathrm{MeV} / \mathrm{c}$, which are used in the particle and spin tracking simulations for the two scenarios, described in Secs. V A and V B.

\begin{tabular}{lcc}
\hline \hline Parameter & Description & Value \\
\hline$N$ & number of particles & $10^{5}$ \\
$q$ & deuteron charge & $1.602 \times 10^{-19} \mathrm{C}$ \\
$m$ & deuteron mass & $3.344 \times 10^{-27} \mathrm{~kg}$ \\
$G$ & deuteron $G$-factor & -0.143 \\
$c$ & speed of light & $2.998 \times 10^{8} \mathrm{~m} / \mathrm{s}$ \\
$\beta=v / c$ & Lorentz $\beta$ & 0.459 \\
$\epsilon$ & beam emittance & $10^{-6} \mu \mathrm{m}$ \\
$\Delta p / p$ & momentum spread & $10^{-4}$ \\
\hline \hline
\end{tabular}

the two solutions reflects the true performance of the method proposed in this paper.

When the dynamics includes electromagnetic fields that are functions of position, time, or frequency, the stochastic expansion coefficients may evolve as a function of time, position, etc. This adds another level of complexity that the SGM must be able to cope with. As a consequence, the performance criterion in Eq. (32) must be modified to account for position (or other) dependencies as well,

$$
\begin{aligned}
& \epsilon_{\mu}(z)=\left|\frac{\mu[\zeta(z)]-\mu[\hat{\zeta}(z)]}{\mu[\zeta(z)]}\right|, \quad \text { and } \\
& \epsilon_{\sigma}(z)=\left|\frac{\sigma[\zeta(z)]-\sigma[\hat{\zeta}(z)]}{\sigma[\zeta(z)]}\right| .
\end{aligned}
$$

Here, $\zeta$ may refer either to the position, velocity, or spin dependence, and $\hat{\zeta}$ constitutes the corresponding estimated value using the SGM, similar to Eq. (32).

\section{NUMERICAL RESULTS}

Two different simulation scenarios are considered here. In the first scenario, described in Sec. V A, uniform fields are used with realistic particle properties, while in the second scenario (Sec. V B) a realistic beam passing through the numerically computed fields ${ }^{7}$ of an RF Wien filter [5] is evaluated.

\section{A. Uniform fields}

This generic simulation scenario serves as a proof-ofconcept demonstrator for the SGM. $10^{5}$ particles are considered, ${ }^{8}$ with phase-space-distributed initial positions and velocities (as described in [5]) traveling in a uniform electromagnetic

\footnotetext{
${ }^{7}$ Electromagnetic package of CST MWS, Computer Simulation Technology, Microwave Studio, CST AG., Darmstadt, Germany, http://www.cst.com

${ }^{8}$ Due to the limited computational resources to carry out the equivalent $\mathrm{MC}$ simulations, only $10^{5}$ particles have been considered here. It should be noted that the SGM with the same resources can support the simulation of a much larger number of particles.
}

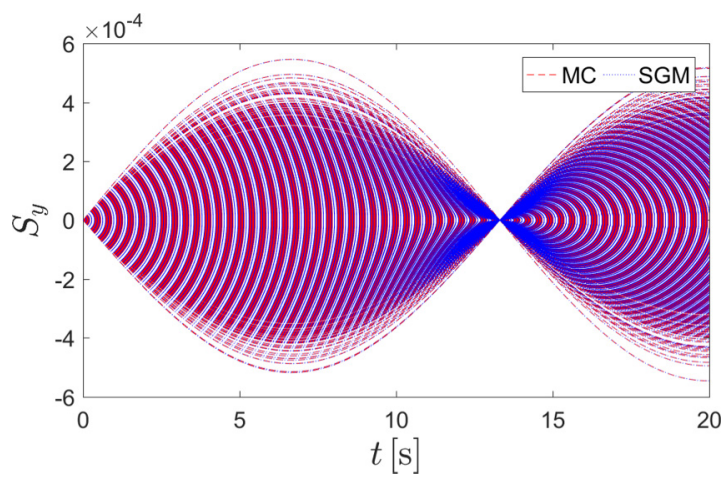

FIG. 3. Result of spin tracking simulations in uniform fields using the MC and the SGM. The horizontal axis shows the time $t$ in seconds, and the vertical axis shows the vertical component $S_{y}(t)$ of the spin vector $\vec{S}(t)$.

field with $\vec{E}=(1,0,0) \mathrm{V} / \mathrm{m}$ and $\vec{H}=(0,1 / 173,0) \mathrm{A} / \mathrm{m},{ }^{9}$ during a time interval from 0 to $20 \mathrm{~s}$.

The positions and transverse angles, $x, x^{\prime}, y$, and $y^{\prime}$, are generated using a $2 \sigma$ beam emittance of $\epsilon_{x, y}=1 \mu \mathrm{m}$. The transverse velocities $v_{x}$ and $v_{y}$ are calculated by multiplying the transverse angles $x^{\prime}$ and $y^{\prime}$ by $v_{z} . v_{z}$ itself is also modeled as a Gaussian random variable with a mean value of $\beta \times c \mathrm{~m} / \mathrm{s}$ and a standard deviation that corresponds to a variation of the beam momentum of $\Delta p / p=10^{-4}$. The beam parameters used in the simulations are summarized in Table I.

Figure 2 shows the tracking results of the $\mathrm{MC}$ and the SGM-based simulation for $x, v_{x}, y$, and $v_{y}$. Due to the extended phase-space distribution of the beam, the transverse velocities $v_{x}$ and $v_{y}$ do not vanish. The particles propagate with roughly half the speed of light for a period of $20 \mathrm{~s}$ in a weak guiding field. Therefore, large position deviations occur, as indicated in panels (a) and (b). In the $x$ direction, this leads to a transverse Lorentz force and an oscillation of the particles around the beam direction, while in the $y$ direction the particles are simply drifting. It was verified by simulations that a beam of vanishing emittance $\epsilon$ and momentum variation $\Delta p / p$ performs a perfect drift motion in both the $x$ and $y$ directions.

Very good agreement between the MC method and the SGM-based simulations can clearly be observed in Fig. 2. In particular, no difference between the oscillation periods and the positions $x$ and velocities $v_{x}$ as a function of time are observed. In terms of position $y$ and velocity $v_{y}$, as depicted in Fig. 2(d), SGM and MC both indicate a pure drift motion.

The stochastic discretization of the T-BMT equation has been implemented as well, and the numerical results are shown in Fig. 3. The fields, despite the fact that they are uniform, can be expanded to include the effects of undesired physical phenomena, such as displacements or rotations. The simulation scenario assumes a horizontally polarized deuteron beam with initial spin vectors in the horizontal (ring) plane, such that $\vec{S}=(1,0,0)$. While the system of ODEs is solved for $S_{x}, S_{y}$, and $S_{z}$, only the vertical spin component $S_{y}$ is displayed in Fig. 3. Figure 3 clearly shows that both the

\footnotetext{
${ }^{9}$ In vacuum, $\vec{B}=\mu_{0} \vec{H}=4 \times \pi \times 10^{-7} \vec{H}$.
} 


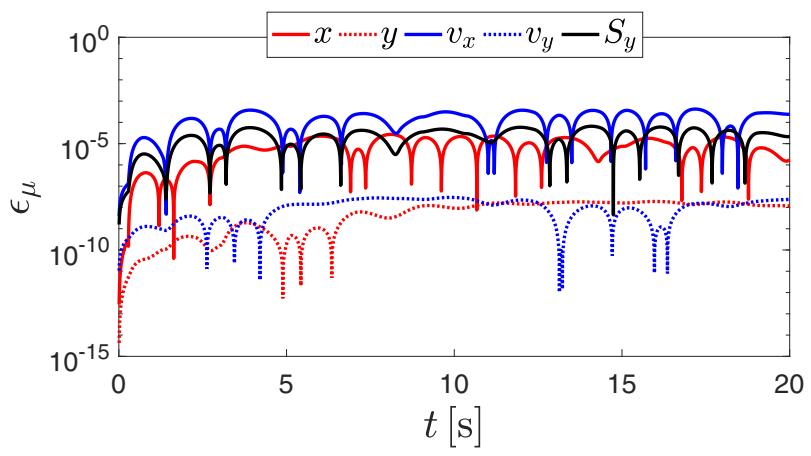

(a)

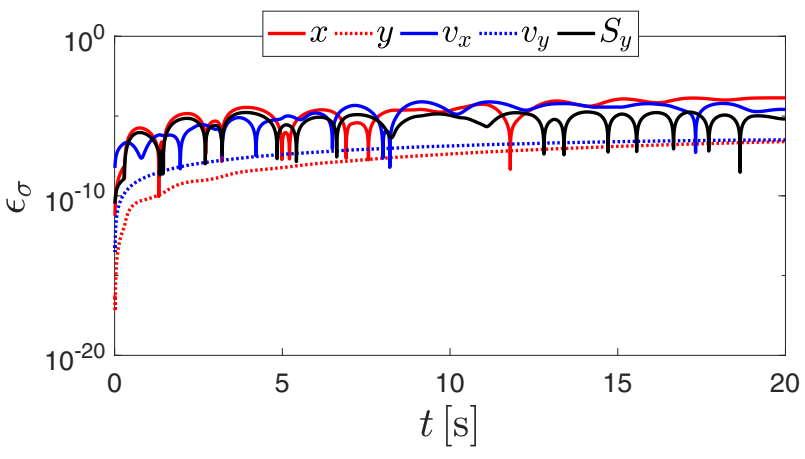

(b)

FIG. 4. (a) The error analysis of the uniform field scenario (Sec. V A) of the mean value $\epsilon_{\mu}(t)$ using Eq. (32a), and (b) the standard deviation $\epsilon_{\sigma}(t)$ using Eq. (32b) for the quantities $x, y, v_{x}, v_{y}$, and $S_{y}$ in the time interval from $t=0$ to $20 \mathrm{~s}$.

oscillation magnitude and the frequency of MC and SGM evolve synchronously.

By applying Eq. (32), the performance of the SGM is demonstrated in Figs. 4(a) and 4(b). As we are dealing with stochastic quantities, the mean and standard deviations are used as performance indicators. All estimated SGM solutions $\left(\hat{x}, \hat{v_{x}}, \hat{y}, \hat{v_{y}}\right.$, and $\left.\hat{S_{y}}\right)$ do not deviate by more than $10^{-5}$ from the MC solutions $\left(x, v_{x}, y, v_{y}\right.$, and $\left.S_{y}\right)$.

\section{B. Tracking with realistic RF Wien filter fields}

In this section, we present simulation results using the MC and the SGM, where both methods were applied to a deuteron beam (see Table I) traveling along the $z$ axis of an RF Wien filter, whose electromagnetic fields were calculated using a full-wave simulation based on $\mathrm{CST}^{7}$. A detailed description of the calculations and the field distributions can be found in [5].

The waveguide RF Wien filter constitutes a novel device that is presently installed at COSY-Jülich. The aim is to perform a first direct measurement of the deuteron EDM [1]. The device is characterized by high-quality electromagnetic fields that, when properly matched, provide a vanishing Lorentz force. This is achieved by adjusting the field quotient $Z_{q},{ }^{10}$ and this makes the device transparent to the passage of particles [see Eq. (3) of [5]].

At the location where the device is installed at COSY, the beam size is adjustable by modifying the $\beta$ function to values

\footnotetext{
${ }^{10}$ The field quotient is defined as the ratio of total electric field to total magnetic field.
}

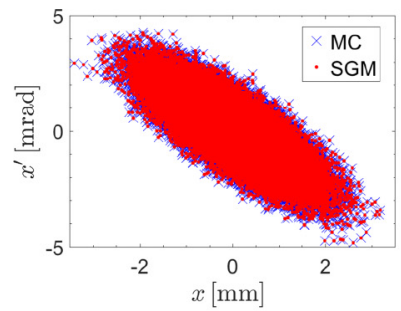

(a)

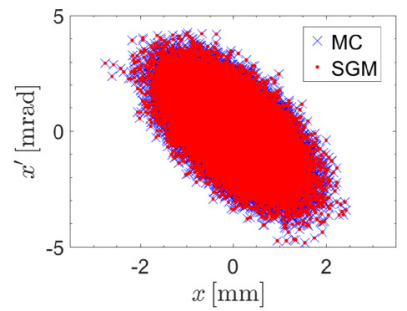

(b)

between about $\beta=0.4$ and $4 \mathrm{~m}$ [4]. In this paper, we consider only the case when $\beta=0.4$. A typical initial phase-space ellipse for a well-cooled beam at the entrance of the RF Wien filter at $z=1 \mathrm{~mm}$ is shown in panel (a) of Fig. 5 (for other beam properties, see also Table I). In panels (b)-(d), the phase-space ellipses are displayed at other positions inside the RF Wien filter at $z=210,610$, and $810 \mathrm{~mm}$. In all cases, SGM and MC results are in very good agreement.

The simulation results from the MC and SGM of the trajectories in the $x z$ plane of the drift region inside the RF Wien filter are shown in Fig. 6. The MC results are represented by the dashed red lines, and the SGM simulations by the dotted red lines. Evidently, also here, the SGM results perfectly coincide with their MC counterparts.

Using Eqs. (33a) and (33b), the deviation of the simulation results of the SGM and the corresponding MC are quantified and depicted in Fig. 7. The horizontal axis represents the beam axis in the RF Wien filter. The solid lines denote the errors calculated by considering the mean values $\epsilon_{\mu}(z)$ with respect to the horizontal position $x$ and the velocity $v_{x}$ in the $x$ direction, while the dashed lines correspond to the errors related to the standard deviation $\epsilon_{\sigma}(z)$ for the same quantities. The relative deviation of $x_{\epsilon_{\mu}}$ and $v_{x_{\epsilon \mu}}$ does not exceed $10^{-7}$, while that of $x_{\epsilon_{\sigma}}$ and $v_{x_{\sigma}}$ remains below $10^{-10}$. The smallness of the calculated errors indicates the excellent performance of the SGM.

\section{Comparison of simulation times}

One additional performance criterion besides the error analysis is the comparison of the required simulation times. In the following, the generic scenario of Sec. V A is discussed, with particles properties as listed in Table I.

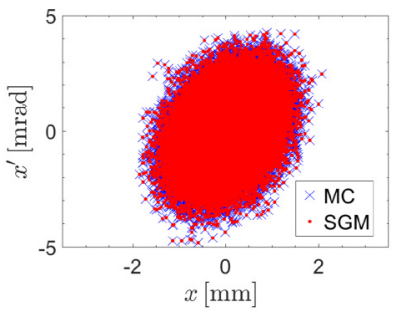

(c)

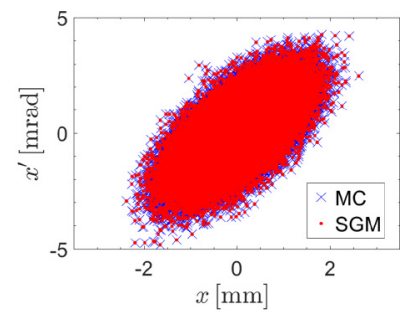

(d)

FIG. 5. Comparison between the phase-space ellipses of the MC tracking results (blue crosses) and the SGM (red dots) along the beam direction in the waveguide RF Wien filter at $z=1$ (a), 210 (b), 610 (c), and $810 \mathrm{~mm}$ (d) [5] (Sec. V B). 


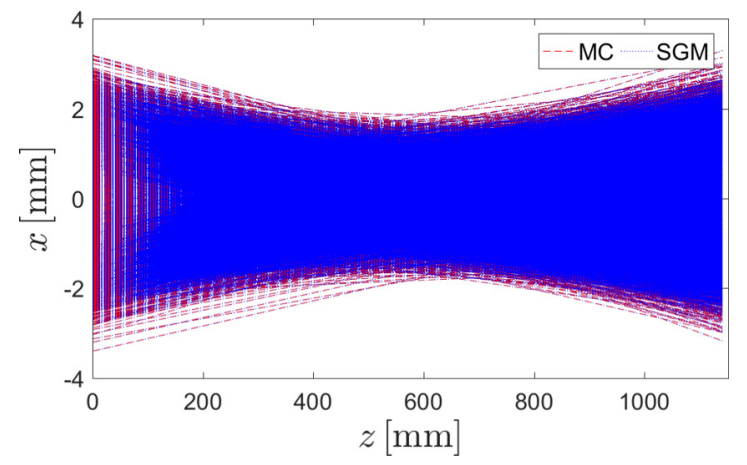

FIG. 6. Trajectory simulation of the $x z$ plane in the drift region along the RF Wien filter (Sec. V B) using the MC and the SGM.

The MC simulations require the evaluation of the system of beam and spin ODEs [Eqs. (5) and (6)]. The MATLAB simulation environment provides a powerful vectorization option that has been used here to parallelize the execution of the code. As shown in Fig. 8, for fewer than $N \approx 10^{5}$ particles, $\mathrm{MC}$ and SGM are about equally fast, but when $N$ increases further, the required time for the $\mathrm{MC}$ increases exponentially, ${ }^{11}$ while the corresponding time required for the SGM stays about constant.

Regardless of the number of particles, the system of ODEs involved in the SGM is evaluated exactly $P$ times, ${ }^{12}$ corresponding to the number of basis functions. This is the main reason why the SGM is so much faster than the MC, without reduction in accuracy, as evidenced in Figs. 4(a), 4(b), and 7. When the expansion order $p$ is kept constant, the number of basis functions remains constant as well, and hence also the simulation time $T_{s}=4.14 \pm 0.39 \mathrm{~s}$, as shown in Fig. 8 .

\footnotetext{
${ }^{11}$ The time required for the simulation of $2 \times 10^{6}$ particles on the available machine ${ }^{6}$ amounted to $925.42 \pm 0.82 \mathrm{~s}$. Turning off the vectorization option, tracking up to $10^{9}$ particles becomes possible, but the simulation time becomes prohibitively large.

${ }^{12}$ Even this number can be further reduced using a sparse version of PCE [11].
}

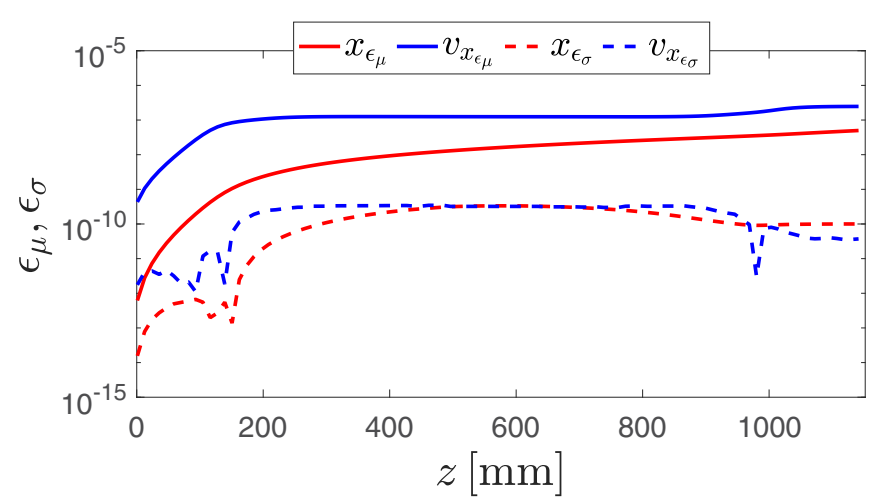

FIG. 7. Error analysis involving the mean value $\epsilon_{\mu}(z)$ and the standard deviation $\epsilon_{\sigma}(z)$ of the quantities $x$ and $v_{x}$ along the beam direction inside the waveguide RF Wien filter (Sec. VB).

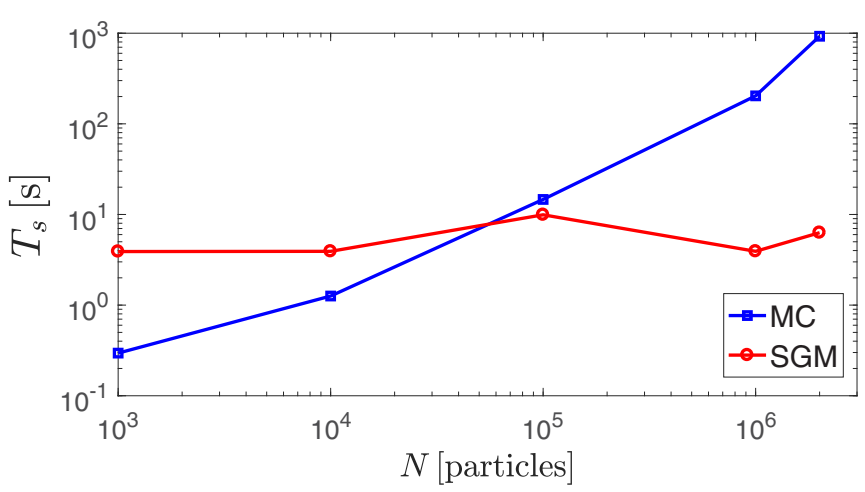

FIG. 8. Comparison of the simulation time required for the parallelized MC and the SGM. For particle numbers below about $10^{5}$, the methods are comparable. For larger particle numbers with a constant expansion order of $p=4$, the time required for the SGM stays constant, while the demand for the MC increases exponentially.

\section{CONCLUSION}

This paper reports on the application of the stochastic Galerkin method (SGM) to beam- and spin-tracking simulations. The method has been shown to work well with uniform fields. We have also applied it to a realistic scenario, involving the electromagnetic fields of a waveguide RF Wien filter, and also in this case the results indicate very good agreement with the Monte Carlo (MC) simulations, which were carried out concurrently.

The error calculations carried out show that the performance of the SGM is statistically equivalent to the MC method, but with much lower computational demand. While the computational effort of MC-based simulations increases exponentially as a function of particle number, the computational effort involved in the SGM stays constant, independent of the number of tracked particles. The SGM transforms the original system of beam and spin ODEs into an augmented system of chaos coefficients, which are determined and then used to reconstruct the response for an arbitrary number of particles. The SGM is therefore capable of tracking large particle numbers in a short time without compromising on the accuracy, and in this way it provides a very efficient yet accurate alternative to MC-based methods.

A potential future application of the SGM might be spin-tracking calculations for storage rings, which are necessary in particular for precision experiments, such as the search for electric dipole moments. In such cases, an ultimate precision is required in the presence of uncertainties of the optical elements that constitute the machine. The SGM allows one to conveniently take into account systematic errors from different sources and to build a hierarchy of error sources. In view of the computational effort required for the MC-based error evaluation, the SGM may thus become an indispensable tool for future precision experiments.

\section{ACKNOWLEDGMENTS}

This work has been performed in the framework of the JEDI collaboration and is supported by an ERC AdvancedGrant of the European Union (Proposal No. 694340). We 
would like to thank Alexander Nass, Helmut Soltner, Martin Gaisser, Jörg Pretz, Andreas Lehrach, and Jan Hetzel for their useful comments and a careful reading of the manuscript.
[1] F. Rathmann, A. Saleev, N. N. Nikolaev, and on behalf of the JEDI and srEdm Collaborations, J. Phys.: Conf. Ser. 447, 012011 (2013).

[2] W. M. Morse, Y. F. Orlov, and Y. K. Semertzidis, Phys. Rev. ST Accel. Beams 16, 114001 (2013).

[3] R. Maier, Nucl. Instrum. Methods Phys. Res., Sect. A 390, 1 (1997).

[4] C. Weidemann, F. Rathmann, H. J. Stein, B. Lorentz, Z. Bagdasarian, L. Barion, S. Barsov, U. Bechstedt, S. Bertelli, D. Chiladze, G. Ciullo, M. Contalbrigo, S. Dymov, R. Engels, M. Gaisser, R. Gebel, P. Goslawski, K. Grigoriev, G. Guidoboni, A. Kacharava, V. Kamerdzhiev, A. Khoukaz, A. Kulikov, A. Lehrach, P. Lenisa, N. Lomidze, G. Macharashvili, R. Maier, S. Martin, D. Mchedlishvili, H. O. Meyer, S. Merzliakov, M. Mielke, M. Mikirtychiants, S. Mikirtychiants, A. Nass, N. N. Nikolaev, D. Oellers, M. Papenbrock, A. Pesce, D. Prasuhn, M. Retzlaff, R. Schleichert, D. Schröer, H. Seyfarth, H. Soltner, M. Statera, E. Steffens, H. Stockhorst, H. Ströher, M. Tabidze, G. Tagliente, P. T. Engblom, S. Trusov, Y. Valdau, A. Vasiliev, and P. Wüstner, Phys. Rev. ST Accel. Beams 18, 020101 (2015).

[5] J. Slim, R. Gebel, D. Heberling, F. Hinder, D. Hölscher, A. Lehrach, B. Lorentz, S. Mey, A. Nass, F. Rathmann, L. Reifferscheidt, H. Soltner, H. Straatmann, F. Trinkel, and J. Wolters, Nucl. Instrum. Methods Phys. Res., Sect. A 828, 116 (2016).

[6] A. Saleev, N. N. Nikolaev, F. Rathmann, W. Augustyniak, Z. Bagdasarian, M. Bai, L. Barion, M. Berz, S. Chekmenev, G. Ciullo, S. Dymov, D. Eversmann, M. Gaisser, R. Gebel, K. Grigoryev, D. Grzonka, G. Guidoboni, D. Heberling, V. Hejny, N. Hempelmann, J. Hetzel, F. Hinder, A. Kacharava, V. Kamerdzhiev, I. Keshelashvili, I. Koop, A. Kulikov, A. Lehrach, P. Lenisa, N. Lomidze, B. Lorentz, P. Maanen, G. Macharashvili, A. Magiera, D. Mchedlishvili, S. Mey, F. Müller, A. Nass, A. Pesce, D. Prasuhn, J. Pretz, M. Rosenthal, V. Schmidt, Y. Semertzidis, Y. Senichev, V. Shmakova, A. Silenko, J. Slim, H. Soltner, A. Stahl, R. Stassen, E. Stephenson, H. Stockhorst, H. Ströher, M. Tabidze, G. Tagliente, R. Talman, P. T. Engblom, F. Trinkel, Y. Uzikov, Y. Valdau, E. Valetov, A. Vassiliev, C. Weidemann, A. Wrońska, P. Wüstner, P. Zuprański, and M. Zurek (JEDI Collaboration), Phys. Rev. Accel. Beams 20, 072801 (2017).

[7] E. M. Metodiev et al., Nucl. Instrum. Methods A 797, 311 (2015).

[8] M. S. Rosenthal, Ph.D. thesis, RWTH Aachen University, 2016.

[9] V. Anastassopoulos, S. Andrianov, R. Baartman, S. Baessler, M. Bai, J. Benante, M. Berz, M. Blaskiewicz, T. Bowcock, K. Brown, B. Casey, M. Conte, J. D. Crnkovic, N. D'Imperio, G. Fanourakis, A. Fedotov, P. Fierlinger, W. Fischer, M. O. Gaisser, Y. Giomataris, M. Grosse-Perdekamp, G. Guidoboni, S. Hacıömeroğlu, G. Hoffstaetter, H. Huang, M. Incagli, A. Ivanov, D. Kawall, Y. I. Kim, B. King, I. A. Koop, D. M. Lazarus, V. Lebedev, M. J. Lee, S. Lee, Y. H. Lee, A. Lehrach, P. Lenisa, P. L. Sandri, A. U. Luccio, A. Lyapin, W. MacKay, R. Maier,
K. Makino, N. Malitsky, W. J. Marciano, W. Meng, F. Meot, E. M. Metodiev, L. Miceli, D. Moricciani, W. M. Morse, S. Nagaitsev, S. K. Nayak, Y. F. Orlov, C. S. Ozben, S. T. Park, A. Pesce, E. Petrakou, P. Pile, B. Podobedov, V. Polychronakos, J. Pretz, V. Ptitsyn, E. Ramberg, D. Raparia, F. Rathmann, S. Rescia, T. Roser, H. K. Sayed, Y. K. Semertzidis, Y. Senichev, A. Sidorin, A. Silenko, N. Simos, A. Stahl, E. J. Stephenson, H. Ströher, M. J. Syphers, J. Talman, R. M. Talman, V. Tishchenko, C. Touramanis, N. Tsoupas, G. Venanzoni, K. Vetter, S. Vlassis, E. Won, G. Zavattini, A. Zelenski, and K. Zioutas, Rev. Sci. Instrum. 87, 115116 (2016).

[10] F. Augustin and P. Rentrop, Numer. Math. 122, 399 (2012).

[11] J. Slim, F. Rathmann, A. Nass, H. Soltner, R. Gebel, J. Pretz, and D. Heberling, Nucl. Instrum. Methods Phys. Res., Sect. A 859, 52 (2017).

[12] D. Xiu and G. E. Karniadakis, SIAM J. Sci. Comput. 24, 619 (2002).

[13] P. Parthasarathy and R. Sudhesh, Appl. Math. Lett. 19, 1083 (2006).

[14] G. Blatman and B. Sudret, Probab. Eng. Mech. 25, 183 (2010).

[15] W. Yu, W. Li, A. Elsherbeni, and Y. Rahmat-Samii, Advanced Computational Electromagnetic Methods, Artech House Antennas and Electromagnetics Analysis Library (Artech House, Norwood, 2015).

[16] M. Abramowitz, Handbook of Mathematical Functions, With Formulas, Graphs, and Mathematical Tables (Dover, New York, 1970).

[17] O. P. Le Maître and O. M. Knio, Spectral Methods for Uncertainty Quantification: With Applications to Computational Fluid Dynamics, Scientific Computation (Springer, Dordrecht, 2010).

[18] P. Offermann, H. Mac, T. T. Nguyen, S. Clénet, H. D. Gersem, and K. Hameyer, IEEE Trans. Magn. 51, 1 (2015).

[19] O. Zienkiewicz, R. Taylor, and J. Zhu, The Finite Element Method: Its Basis and Fundamentals (Elsevier, Oxford, 2013).

[20] J. Jin, The Finite Element Method in Electromagnetics, 3rd ed. (Wiley, Hoboken, 2014).

[21] J. Bittencourt, Fundamentals of Plasma Physics (Springer, New York, 2004).

[22] D. Xiu, Numerical Methods for Stochastic Computations: A Spectral Method Approach (Princeton University Press, Princeton, 2010).

[23] T. Sullivan, Introduction to Uncertainty Quantification, Texts in Applied Mathematics (Springer, Cham, 2015).

[24] P. Pettersson, G. Iaccarino, and J. Nordström, J. Comput. Phys. 257, 481 (2014).

[25] V. Bargmann, L. Michel, and V. L. Telegdi, Phys. Rev. Lett. 2, 435 (1959).

[26] T. Fukuyama and A. J. Silenko, Int. J. Mod. Phys. A 28, 1350147 (2013).

[27] J. Hurtado, Structural Reliability: Statistical Learning Perspectives, Lecture Notes in Applied and Computational Mechanics (Springer, Berlin, 2013). 
[28] Z. Kotulski and W. Szczepinski, Error Analysis with Applications in Engineering, Solid Mechanics and Its Applications (Springer, The Netherlands, 2009).

[29] R. Lebrun and A. Dutfoy, Probab. Eng. Mech. 24, 172 (2009).

[30] G. Cottrill and F. Harmon, Hybrid Gauss pseudospectral and generalized polynomial chaos algorithm to solve stochastic trajectory optimization problems, in AIAA Guidance, Navigation, and Control Conference (American Institute of Aeronautics and Astronautics, Reston, VA, 2011).

[31] M. Gerritsma, J.-B. van der Steen, P. Vos, and G. Karniadakis, J. Comput. Phys. 229, 8333 (2010). 